\title{
Stability of spherically trapped three-dimensional Bose-Einstein condensates against macroscopic fragmentation
}

\author{
Philipp Bader ${ }^{1}$ and Uwe R. Fischer ${ }^{2}$ \\ ${ }^{1}$ Instituto de Matemática Multidisciplinar, Universitat Politècnica de València, E-46022 Valencia, Spain \\ ${ }^{2}$ Center for Theoretical Physics, Department of Physics and Astronomy, Seoul National University, 151-747 Seoul, Korea
}

(Received 22 November 2012; published 28 February 2013)

\begin{abstract}
We consider spherically trapped Bose gases in three dimensions with contact interactions and investigate whether the Bose-Einstein condensate at zero temperature is stable against macroscopic fragmentation into a small number of mutually incoherent pieces. Our results are expressed in terms of a dimensionless interaction measure proportional to the Thomas-Fermi parameter. It is shown that while three-dimensional condensates are inherently much more stable against macroscopic fragmentation than their quasi-one- and quasi-two-dimensional counterparts, they fragment at a sufficiently large value of the dimensionless interaction measure, which we determine both fully numerically and semianalytically from a continuum limit of large particle numbers.
\end{abstract}

DOI: 10.1103/PhysRevA.87.023632

PACS number(s): $03.75 . \mathrm{Gg}$

\section{INTRODUCTION}

Bose-Einstein condensation [1,2] of noninteracting bosons can in principle occur in an arbitrarily large spatial dimension $D$, depending on both the properties of the single-particle spectrum and the confining potential [3]. In contrast, the Hohenberg-Mermin-Wagner theorem rules out, independently of the strength of interactions, Bose-Einstein condensation in $D \leqslant 2[4,5]$. The latter theorem, however, applies to homogeneous condensates in the thermodynamic limit, where longrange phase fluctuations trigger the decay of the Bose-Einstein condensates into infinitely many fragments. This changes for trapped condensates, where the finite extension of the gas cuts off the phase fluctuations in the corresponding directions. While an explicitly interaction-independent formulation of the theorem is still possible, the geometric shape of the condensate enters the Bogoliubov inequality on which the Hohenberg-Mermin-Wagner theorem rests [6].

It is well known that in three spatial dimensions, fragmentation does not occur in the thermodynamic limit and in a homogeneous system for positive interaction coupling [7,8], while for a negative coupling constant the system is unstable. For a trapped, that is, spatially localized, and inhomogeneous system in three dimensions, the relevant dimensionless parameter to measure the importance of interactions over the singleparticle kinetic and trapping contributions to the energy is the Thomas-Fermi parameter $N a_{s} / l_{0}$ (where $a_{s}$ and $l_{0}$ are $s$-wave scattering and the harmonic trapping length, respectively). It was previously observed by us that harmonic trapping and positive interaction coupling can lead to fragmentation into two mutually incoherent macroscopic pieces, forming a so-called fragmented condensate, well before the thermodynamic limit is taken for quasi-one-dimensional (quasi-1D) and quasi-2D gases $[9,10]$. To more completely elucidate the dimensional dependence of the many-body physics of fragmentation, we present here a detailed analysis of fragmentation for the completely symmetric example of a spherically trapped 3D condensate. Viewed from a different angle, we investigate to what extent the conventional textbook wisdom [11,12], that when the Thomas-Fermi parameter of a 3D spherically trapped condensate is going to infinity yields a interaction-dominated single Bose-Einstein condensate (with a parabolic shape in this Thomas-Fermi limit and in a harmonic trap), needs revision.

In three dimensions, due to the spherical symmetry of the system, even when the field operator expansion is restricted to the low-energy sector, there are potentially four singleparticle states that are macroscopically occupied. By numerical analysis and general symmetry arguments, we find that fragmentation is dominated by two orbitals at a dimensionless coupling measure that is proportional to the Thomas-Fermi parameter. The critical coupling measure is one (two) order (orders) of magnitude larger than the corresponding measure in the quasi-2D (quasi-1D) cases. In addition, the maximal degree of fragmentation [9] turns out to be significantly smaller than in the latter cases. Our result therefore implies the rapidly growing persistence of an interacting trapped scalar Bose-Einstein condensate against macroscopic fragmentation upon increasing the spatial dimension.

\section{SPHERICALLY TRAPPED GASES IN THREE DIMENSIONS}

\section{A. Four-mode approximation for the Hamiltonian}

To facilitate a comparison with the previously treated quasi1D and quasi-2D trapping cases, we will make a one-parameter variational ansatz for the single-particle orbitals as in Ref. [6]. This involves ground and first excited states of the harmonic oscillator, with the variational parameter chosen to be the harmonic oscillator length. Compared to fully self-consistent multiconfigurational Hartree calculations as performed, e.g., in Refs. [13-16], while being less quantitatively accurate, the variational approach leads to a qualitatively correct picture of the fragmentation phenomenon. A particular merit of this approach is that the parameter dependence of the fragmentation transition is transparent: We find that fragmentation is decided by a single parameter, $G_{3}$ in Eq. (10) below, that measures the relative importance of interactions over the single-particle (trapping) energies. In addition, the variational approach is capable of dealing with the limit of very large particle numbers $N$; in its continuum limit, which we will derive below, there is indeed no upper bound to the value of $N$. This is particularly beneficial in three spatial dimensions, 
where the particle numbers at the same densities are obviously larger than in one- and two-dimensional systems; we were able to numerically calculate, within relatively short time scales, systems with up to $N \sim 10^{6}$ particles.

To formulate the proper variational orbitals basis, we first write down the well-known eigenstates and energies of the isotropic harmonic oscillator in three spatial dimensions

$$
\begin{aligned}
\psi_{n l m} & =Y_{l}^{m}(\theta, \phi) N_{n l} r^{l} \exp \left[-\frac{r^{2}}{2}\right] L_{n}^{(l+1 / 2)}\left(r^{2}\right), \\
E_{n l} & =\omega\left(2 n+l+\frac{3}{2}\right)
\end{aligned}
$$

where the normalization $N_{n, l}=\frac{\sqrt{\Gamma\left(n+l+\frac{1}{2}\right)}}{\sqrt{n ! \Gamma\left(l+\frac{1}{2}\right)}}$ and the functions $Y_{l}^{m}$ and $L_{n}^{(l+1 / 2)}$ are spherical harmonics and generalized Laguerre polynomials, respectively. The width of the singleparticle basis functions is expressed by a length $R$, which is the scaling length of the radial coordinate $r$, that is, we put $r / R \rightarrow r$. For noninteracting condensates the width $R$ is given by the harmonic oscillator length $R=l_{0}=\omega^{-1 / 2}$ ( $\hbar=M=1$, where $M$ is the boson mass). In the following we assume $R$ to be a variational parameter, which will determine the family of solutions of the many-body equations, i.e., whether single or fragmented condensates are obtained is determined by the variation of $R$. In order to make large particle number calculations feasible, as well as to render the energy landscape of the eigenvalue problem in its most transparent form, the dimensionality of the variational space is reduced by assuming that all single-particle orbitals scale with the same $R$.

According to Eq. (1), the four energetically lowest states are given by the quantum numbers $n=0$ and $l=0$ (ground) and $n=0, l=1$, and $m=-1,0,1$ (first excited) [adopting the Condon-Shortley phase convention for spherical harmonics],

$$
\begin{aligned}
\psi_{0} & \equiv \psi_{000}=\frac{1}{\pi^{3 / 4}} \exp \left[-\frac{r^{2}}{2}\right], \\
\psi_{1} & \equiv \psi_{010}=\sqrt{2} r \cos \theta \psi_{0}(r), \\
\psi_{+} & \equiv \psi_{011}=-r e^{i \phi} \sin \theta \psi_{0}(r), \\
\psi_{-} & \equiv \psi_{01-1}=r e^{-i \phi} \sin \theta \psi_{0}(r) .
\end{aligned}
$$

The contact-interaction many-body Hamiltonian reads, written in terms of the full field operators,

$$
\begin{aligned}
\hat{H}= & \int d^{3} x \hat{\Psi}^{\dagger}(\boldsymbol{x})\left[-\frac{\nabla^{2}}{2}+\frac{1}{2} \omega^{2} r^{2}\right] \hat{\Psi}(\boldsymbol{x}) \\
& +\frac{g}{2} \iint d^{3} x \hat{\Psi}^{\dagger}(\boldsymbol{x}) \hat{\Psi}^{\dagger}(\boldsymbol{x}) \hat{\Psi}(\boldsymbol{x}) \hat{\Psi}(\boldsymbol{x}) .
\end{aligned}
$$

After truncating the field operator expansion, including the four modes (2), we obtain

$$
\begin{aligned}
\hat{H}= & \sum_{i=0,1, \pm}\left[\epsilon_{i} \hat{n}_{i}+\frac{1}{2} C_{i} \hat{n}_{i}\left(\hat{n}_{i}-1\right)\right] \\
& +\frac{1}{2} D_{1} \hat{n}_{0} \hat{n}_{1}+\frac{1}{2} D_{2}\left(\hat{n}_{0} \hat{n}_{+}+\hat{n}_{0} \hat{n}_{-}\right) \\
& +\frac{1}{2} D_{3}\left(\hat{n}_{1} \hat{n}_{+}+\hat{n}_{1} \hat{n}_{-}\right)+\frac{1}{2} D_{4} \hat{n}_{+} \hat{n}_{-}+\frac{1}{2}\left\{E_{1} \hat{a}_{1}^{\dagger} \hat{a}_{1}^{\dagger} \hat{a}_{0} \hat{a}_{0}\right. \\
& \left.+E_{2} \hat{a}_{+}^{\dagger} \hat{a}_{-}^{\dagger} \hat{a}_{0} \hat{a}_{0}+E_{3} \hat{a}_{+}^{\dagger} \hat{a}_{-}^{\dagger} \hat{a}_{1} \hat{a}_{1}\right\}+ \text { H.c. }
\end{aligned}
$$

Note that the pair-exchange scattering (terms proportional to $E_{i}$ ) occurs also between energetically degenerate orbitals (degenerate on the single-particle level), being represented by the term proportional to $E_{3}$ and involving the excited states $m= \pm 1$ and $0(l=1)$. This is distinct from the quasi-1D and quasi-2D cases treated in Ref. [10], where pair-exchange scattering only occurs between pairs of ground and excited single-particle states.

The interaction matrix elements $V_{i j k l}=$ $g \iiint r^{2} \sin \theta d r d \theta d \phi \psi_{i}^{*}(\boldsymbol{r}) \psi_{j}^{*}(\boldsymbol{r}) \psi_{k}(\boldsymbol{r}) \psi_{l}(\boldsymbol{r})$ are related to the coefficients in Eq. (4) as follows. The nonvanishing pair-exchange matrix elements are

$E_{1}=V_{1100}, \quad E_{2}=V_{+-00}+V_{-+00}, \quad E_{3}=V_{+-11}+V_{-+11}$.

The remaining coefficients are of the density-density type,

$$
\begin{aligned}
& C_{0}=V_{0000}, \quad C_{1}=V_{1111}, \quad C_{+}=V_{++++}, \\
& C_{-}=V_{----}, \quad D_{1}=V_{0101}+V_{1010}+V_{1001}+V_{0110}, \\
& D_{2}=D_{1}(1 \rightarrow \pm), \quad D_{3}=D_{1}(0 \rightarrow \pm) \\
& D_{4}=D_{1}(0 \rightarrow+, 1 \rightarrow-) .
\end{aligned}
$$

The result for the scattering coefficients (reinstating now the variational harmonic oscillator length) may be written in a compact notation in the following way:

$$
\begin{aligned}
& \left\{C_{0}, C_{1}, C_{ \pm}, D_{1}, D_{2}, D_{3}, D_{4}, E_{1}, E_{2}, E_{3}\right\} \\
& \quad=\frac{g}{(2 \pi)^{3 / 2} R^{3}}\left\{1, \frac{3}{4}, \frac{1}{2}, 2,4,2,2, \frac{1}{2}, 1, \frac{1}{2}\right\} .
\end{aligned}
$$

The single-particle energies are given by $\epsilon_{i}=$ $\int r^{2} \sin \theta d r d \theta d \phi\left[\left|\nabla \psi_{i}\right|^{2} / 2+\omega^{2} r^{2}\left|\psi_{i}\right|^{2} / 2\right]$ and read

$$
\begin{aligned}
& \epsilon_{0}=\frac{3}{4}\left[\frac{1}{R^{2}}+\omega^{2} R^{2}\right], \quad \epsilon_{1}=\frac{5}{4}\left[\frac{1}{R^{2}}+\omega^{2} R^{2}\right], \\
& \epsilon_{ \pm}=\frac{5}{4}\left[\frac{1}{R^{2}}+\omega^{2} R^{2}\right]=\epsilon_{1} .
\end{aligned}
$$

Defining the scaled variational parameter $\Lambda=R / l_{0}$, we have as the typical units of single-particle energies and coupling constants

$$
\epsilon_{0}=\frac{3}{4} \omega\left(\frac{1}{\Lambda^{2}}+\Lambda^{2}\right), \quad C_{0}=\frac{G_{3} \omega}{N \Lambda^{3}},
$$

where we introduced the dimensionless interaction coupling

$$
G_{3}=\frac{N g}{(2 \pi)^{3 / 2} l_{0}} .
$$

Like its quasi-1D and quasi-2D counterparts $G_{1}=\frac{N g l_{z}}{(2 \pi)^{3 / 2} l_{\perp}^{2}}$ and $G_{2}=\frac{N g}{(2 \pi)^{3 / 2} l_{z}}$, where $l_{z}$ and $l_{\perp}$ are harmonic oscillator lengths of a cylindrical trap, the quantity $G_{3}$ measures the relative importance of the total interaction and the kinetic and potential energy terms in the Hamiltonian. In the present spherically trapped $3 \mathrm{D}$ case, $G_{3}$ is simply directly proportional to the well-known Thomas-Fermi parameter [12], which, as will be demonstrated below, is the single parameter deciding the question of coherence versus fragmentation. 


\section{B. Wave-function ansatz and the eigenvalue problem}

We employ a general four-mode ansatz in the Fock subspace of fixed total particle number

$$
|\Psi\rangle=\sum_{l_{1}, l_{ \pm}} \psi_{l_{1}, l_{+}, l_{-}}\left|N-l_{1}-l_{+}-l_{-}, l_{1}, l_{+}, l_{-}\right\rangle .
$$

The total energy $E=\langle\Psi|\hat{H}| \Psi\rangle$ in terms of the level occupation amplitudes $\psi_{l_{1}, l_{+}, l_{-}}$then reads

$$
\begin{aligned}
E= & c_{l_{1}, l_{ \pm}} \sum_{i=0,1, \pm}\left|\psi_{l_{1}, l_{ \pm}}\right|^{2}+\frac{1}{2} E_{1} d_{1} \psi_{l_{1}, l_{+}, l_{-}}^{*} \psi_{l_{1}+2, l_{+}, l_{-}}+\frac{1}{2} E_{2} d_{2} \psi_{l_{1}, l_{+}, l_{-}}^{*} \psi_{l_{1}, l_{+}+1, l_{-}+1}+\frac{1}{2} E_{3} d_{3} \psi_{l_{1}, l_{+}, l_{-}}^{*} \psi_{l_{1}-2, l_{+}+1, l_{-}+1} \\
& +\frac{1}{2} E_{1} d_{1} \psi_{l_{1}+2, l_{+}, l_{-}}^{*} \psi_{l_{1}, l_{+}, l_{-}}+\frac{1}{2} E_{2} d_{2} \psi_{l_{1}, l_{+}+1, l_{-}+1}^{*} \psi_{l_{1}, l_{+}, l_{-}}+\frac{1}{2} E_{3} d_{3} \psi_{l_{1}-2, l_{+}+1, l_{-}+1}^{*} \psi_{l_{1}, l_{+}, l_{-}},
\end{aligned}
$$

where the diagonal and pair-exchange coefficients take the explicit form

$$
\begin{aligned}
c_{l_{1}, l_{ \pm}}= & \epsilon_{0}\left(N-\sum_{i=1, \pm}\right)+\sum_{i=1, \pm} \epsilon_{i} l_{i}+\frac{1}{2} C_{0}\left(N-\sum_{i=1, \pm} l_{i}\right)\left(N-\sum_{i=1, \pm} l_{i}-1\right)+\frac{1}{2} \sum_{i=1, \pm} C_{i} l_{i}\left(l_{i}-1\right) \\
& +\frac{1}{2} D_{1}\left(N-\sum_{i=1, \pm} l_{i}\right) l_{1}+\frac{1}{2} D_{2}\left(N-\sum_{i=1, \pm} l_{i}\right)\left(l_{+}+l_{-}\right)+\frac{1}{2} D_{3} l_{1}\left(l_{+}+l_{-}\right)+\frac{1}{2} D_{4} l_{+} l_{-}, \\
d_{1}\left(l_{1}, l_{+}, l_{-}\right)= & \sqrt{\left(N-\sum_{i=1, \pm} l_{i}-1\right)\left(N-\sum_{i=1, \pm} l_{i}\right)\left(l_{1}+2\right)\left(l_{1}+1\right)}, \\
d_{2}\left(l_{1}, l_{+}, l_{-}\right)= & \sqrt{\left(N-\sum_{i=1, \pm} l_{i}\right)\left(N-\sum_{i=1, \pm} l_{i}\right)\left(l_{+}+1\right)\left(l_{-}+1\right), \quad d_{3}\left(l_{1}, l_{+}, l_{-}\right)=\sqrt{l_{1}\left(l_{1}-1\right)\left(l_{+}+1\right)\left(l_{-}+1\right)}}
\end{aligned}
$$

Finally, the minimization of the energy functional (12) with respect to $\psi_{l_{1}, l_{+}, l_{-}}^{*}$ gives the eigenequations

$$
\begin{aligned}
E \psi_{l_{1}, l_{+}, l_{-}}= & c_{l_{1}, l_{ \pm}} \psi_{l_{1}, l_{+}, l_{-}}+\frac{E_{1}}{2} d_{1}\left(l_{1}, l_{+}, l_{-}\right) \psi_{l_{1}+2, l_{+}, l_{-}}+\frac{E_{2}}{2} d_{2}\left(l_{1}, l_{+}, l_{-}\right) \psi_{l_{1}, l_{+}+1, l_{-}+1} \\
& +\frac{E_{3}}{2} d_{3}\left(l_{1}, l_{+}, l_{-}\right) \psi_{l_{1}-2, l_{+}+1, l_{-}+1}+\frac{E_{1}}{2} d_{1}\left(l_{1}-2, l_{+}, l_{-}\right) \psi_{l_{1}-2, l_{+}, l_{-}}+\frac{E_{2}}{2} d_{2}\left(l_{1}, l_{+}-1, l_{-}-1\right) \psi_{l_{1}, l_{+}-1, l_{-}-1} \\
& +\frac{E_{3}}{2} d_{3}\left(l_{1}+2, l_{+}-1, l_{-}-1\right) \psi_{l_{1}+2, l_{+}-1, l_{-}-1} .
\end{aligned}
$$

\section{SOLVING THE EIGENVALUE PROBLEM}

\section{A. Decomposition into smaller problems: The $k$ subspaces}

We are facing a high-dimensional eigenvalue problem that is difficult to solve for typical particle numbers because the matrix dimensions scale roughly with $N^{3} \times N^{3}$ when naïvely implemented. However, the assumed orbitals allow for algebraic simplifications, to be explained in what follows, in order to significantly reduce the problem size. We note in this context that in Ref. [17], a thorough analysis of angular momentum many-body states has been performed for attractively interacting and rotating Bose gases.

Observe that only a particular set of couplings between the $l_{1}$ and $l_{ \pm}$terms appears in Eq. (14). To be more specific, only couplings between terms where $l_{+}-l_{-}=$const are allowed as a consequence of the model. Similar to the reduction for a three-mode model in the quasi-2D case treated in Ref. [10], this allows us to partition the eigenvalue problem into $2 N+1$ smaller problems by introducing the notation $\psi_{l_{1}, l_{+}}^{k} \equiv$ $\psi_{l_{1}, l_{+}, l_{-}}$with $-N \leqslant k \equiv l_{-}-l_{+} \leqslant N$ and $\psi=0$ for indices such that $\left|l_{1}+2 l_{+}+k\right|>N$. In terms of the many-body amplitudes with index $k$, the new eigenvalue problem, with the relations we have found for the matrix elements [see Eq. (7)], reads

$$
\begin{aligned}
E \psi_{l_{1}, l_{+}}^{k}= & c_{l_{1}, l_{+}}^{k} \psi_{l_{1}, l_{+}}^{k}+\frac{E_{1}}{2} d_{1}^{k}\left(l_{1}, l_{+}\right) \psi_{l_{1}+2, l_{+}}^{k} \\
& +\frac{E_{1}}{2} d_{1}^{k}\left(l_{1}+2, l_{+}\right) \psi_{l_{1}+2, l_{+}}^{k}+\frac{2 E_{1}}{2} d_{2}^{k}\left(l_{1}, l_{+}\right) \psi_{l_{1}, l_{+}+1}^{k} \\
& +\frac{2 E_{1}}{2} d_{2}^{k}\left(l_{1}, l_{+}+1\right) \psi_{l_{1}, l_{+}+1}^{k}+\frac{E_{1}}{2} d_{3}^{k}\left(l_{1}, l_{+}\right) \psi_{l_{1}+2, l_{+}+1}^{k} \\
& +\frac{E_{1}}{2} d_{3}^{k}\left(l_{1}+2, l_{+}+1\right) \psi_{l_{1}+2, l_{+}+1}^{k} .
\end{aligned}
$$

Since $\epsilon_{-}=\epsilon_{+}$and $C_{+}=C_{-}$, we have $c_{l_{1}, l_{+}, l_{-}}=c_{l_{1}, l_{-}, l_{+}}$and $d_{1}, d_{2}, d_{3}$ are also symmetric with respect to interchanging $l_{+}$ and $l_{-}$.

The eigenvalue problem (14) then becomes completely symmetric in $l_{+}$and $l_{-}$, i.e., interchanging them leaves the equation unaltered and we can assume them, backed up with numerical simulations, to be identical for the many-body ground state, up to a global phase $\phi$ in the amplitudes, that is,

$l_{+} \equiv l_{-} \quad$ or formally $\quad \forall l_{1}, l_{ \pm}: \psi_{l_{1}, l_{+}, l_{-}}=e^{i \phi} \psi_{l_{1}, l_{-}, l_{+}}$. 
Physically, this implies an overall zero angular momentum for the ground state, as expected for our fully symmetric setup with repulsive interaction.

We can immediately deduce from Eq. (15) that the ground state at $k=0$ will be (nearly) degenerate due to the decoupling of even and odd values of $l_{1}$. The problem can thus be split further to separate the practically degenerate eigenstates (up to the energy of one particle) that belong to only even or only odd occupation numbers $l_{1}$. These substantial size reductions allow us to solve the eigenvalue problem numerically and yield a matrix size of approximately $N^{2} \times N^{2}$. Due to the coupling structure of the Hamiltonian, this matrix is very sparse and the total number of nonzero entries grows only quadratically with the particle number $O\left(N^{2}\right)$.

We remark that the even-odd degeneracy allows for the free choice of a phase parameter $\theta$ in the superposition of the degenerate eigenstates [18], which depends on the preparation of the state; $\theta \equiv 0$ in what follows.

\section{B. Numerical results}

The ground states for different configurations $N, G_{3}$ have been computed numerically by finding local minima in the energy curve along the variational parameter $\Lambda$. Numerical calculations for particle numbers up to $N=20000$ confirm that $k>0$ states correspond to increasingly higher energies, with the lowest difference ( $k=0$ to $k=1$ ) in energy per particle being approximately $O\left(G_{3}^{0.44} / N \omega\right)$ and relative energy differences $1-E_{k=1} / E_{k=0}$ also scaling with $O(1 / N)$.
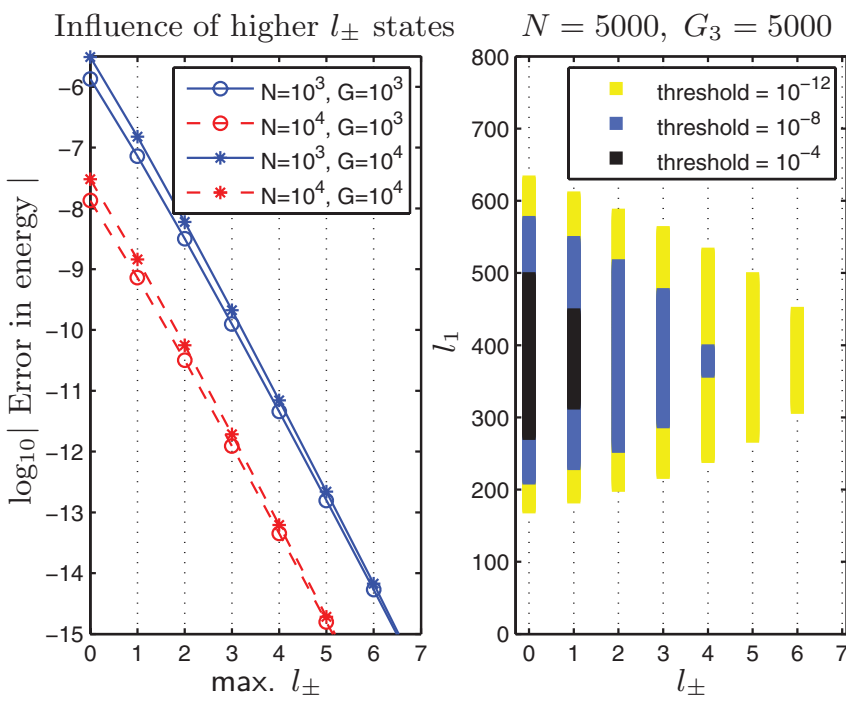

FIG. 1. (Color online) The left panel shows the error committed by truncation of the eigenvalue problem at a fixed value $\max l_{ \pm}$for different values of the particle number $N$ and interaction strength $G_{3}$ with $\Lambda_{\text {min }}$ fixed at the minimal variational energy configuration. Curves with the same line style and symbol correspond to the same $N$ or $G_{3}$, respectively. The right plot visualizes the locations of amplitudes $\left|\psi_{l_{1}, l_{+}}^{0}\right|^{2}$ larger than a given threshold. Note the scaling on the $l_{1}$ axis, which is of $O(N)$, whereas the horizontal axis ends at $l_{ \pm}=7$.
Fixing $k$ at zero and hence $l_{+}=l_{-}$, the occupation of the circulating orbitals stays $\sum_{l_{1},\left(l_{ \pm}>0\right)}\left|\psi_{l_{1}, l \pm}\right|^{2}<3 \%$, with all significant amplitudes located at $l_{ \pm} \sim O(1)$, whereas the occupation of the radially symmetric orbital at $l_{ \pm}=0$ is scaling with $l_{1} \sim O(N)$ (see Fig. 1). Including as few as eight circular states is sufficient to reach machine accuracy for all reasonable configurations $N, G_{3}$.

For large values of $G_{3}$ a second shallow minimum appears in the energy landscape, analogous to the quasi-1D and quasi$2 \mathrm{D}$ cases [10], and the condensate starts to fragment. We have determined numerically that the onset of fragmentation is determined by a critical value of the interaction parameter $\left(G_{3}\right)_{c}$, which depends on particle number. For small $N \sim 1000$, the critical interaction strength is $\left(G_{3}\right)_{c} \approx 5600$, a value that decreases quickly to its asymptotic value $\left(G_{3}\right)_{c}=2480 \pm 10$ for $N=50000$. For large values of $G_{3}$, the fragmented local minimum becomes a global one and the nonfragmented minimum becomes very shallow.

A finite particle number effect on the fragmentation can be observed by varying $N$ for given $G_{3}$ and is illustrated in Fig. 2. For small particle numbers, corresponding to a subcritical $G_{3}(N)$, only one minimum exists and its degree of fragmentation, defined by $\mathcal{F}=1-\left|\lambda_{1}-\lambda_{2}\right| / N$, where $\lambda_{i}$ are the (macroscopic) eigenvalues of the single-particle density matrix [9], rapidly approaches zero when $N$ is increased. Once we have passed the critical value for $N$, fragmentation sets in, with the appearance of a new local energy minimum at a smaller extension $\Lambda$, and quickly approaches a limit that depends on the chosen value of $G_{3}$.

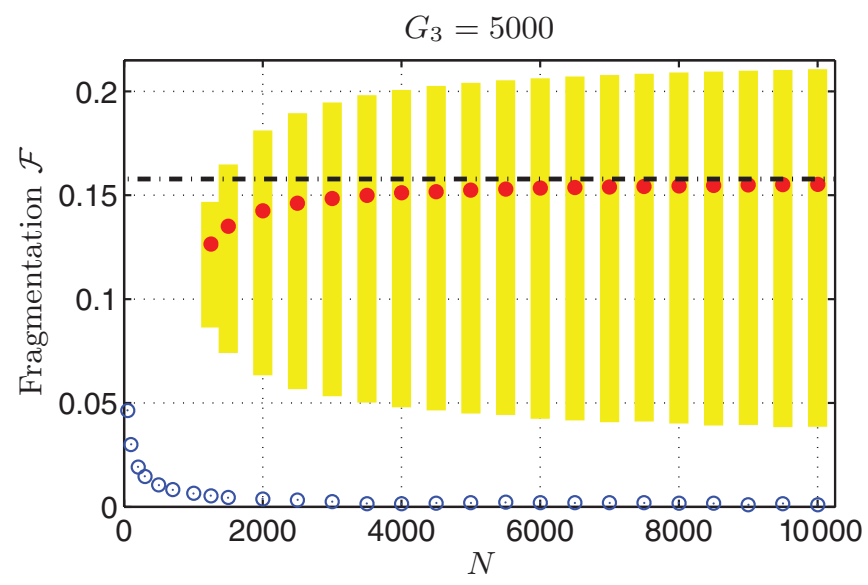

FIG. 2. (Color online) Degree of fragmentation (circles) of the ground state for varying $N$ at fixed $G_{3}=5000$ for nonfragmented (blue open circles) and fragmented (red solid circles) states. Small- $N$ effects include a nonvanishing fragmentation that quickly approaches 0 . After passing a critical $N$, a new minimum appears and asymptotically approaches its maximum for moderate values of $N$. The dash-dotted black line shows the degree of fragmentation at $\mathcal{F}=0.16$, computed in the large- $N$ limit (26). Vertical bars (yellow) indicate the sensitivity of the degree of fragmentation when we allow for an energy variation away from the local minimum via $\Lambda$, up to the energy barrier separating the two minima. 


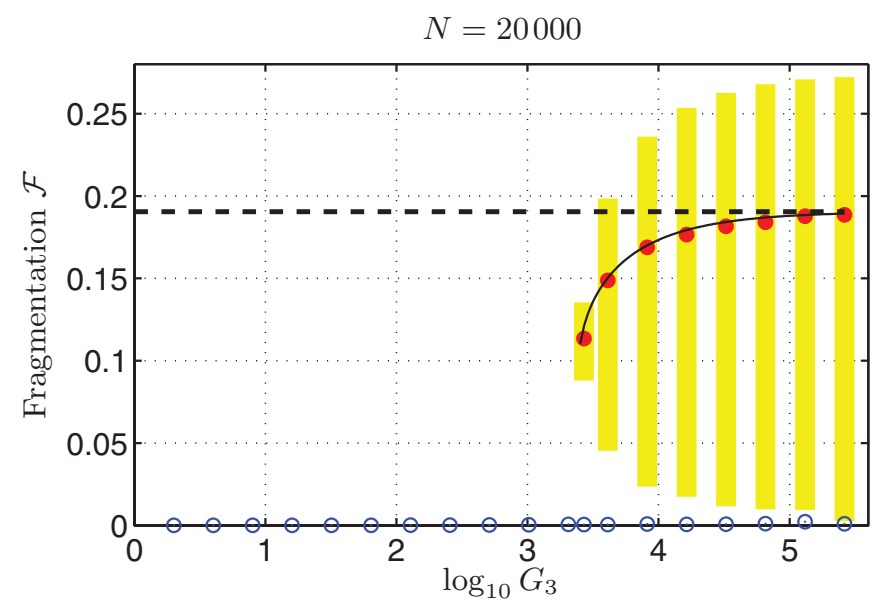

FIG. 3. (Color online) Dependence of degree of fragmentation (circles) on $G_{3}$ for fixed $N=20000$. The single existing minimum (blue open circles) for subcritical $G_{3}$ shows no fragmentation, whereas the one created after a critical $G_{3}$ has been passed (red solid circles) soon asymptotically approaches to its limiting value $\mathcal{F}=0.19$ indicated by the dashed line. The solid line corresponds to the continuum limit results computed via the minimization of Eq. (26).

The dependence of the fragmentation on the interaction strength $G_{3}$ is depicted in Fig. 3. Note the onset of fragmentation after passing the critical $\left(G_{3}\right)_{c}(N)=O\left(10^{3}\right)$.

The above considerations about the low occupancy of circulating states (see Fig. 1) allow us to simplify the problem by neglecting the small $l_{ \pm}>0$ contribution. This yields a two-mode model, for which we apply a continuum limit [19] in the following section.

\section{CONTINUUM LIMIT OF THE EFFECTIVE TWO-MODE MODEL}

\section{A. Derivation of the Schrödinger equation for the mode population}

From the full numerical analysis, we are led to conclude that $l_{ \pm} \neq 0$ is approximately unpopulated and after deletion of the $l_{ \pm}>0$ terms, the eigenvalue problem (15) reduces to

$$
\begin{aligned}
E \psi_{l_{1}, 0}^{k}= & c_{l_{1}, 0} \psi_{l_{1}, 0}^{k}+\frac{E_{1}}{2} d_{1}\left(l_{1}, 0\right) \psi_{l_{1}+2,0}^{k} \\
& +\frac{E_{1}}{2} d_{1}\left(l_{1}-2,0\right) \psi_{l_{1}-2,0}^{k} .
\end{aligned}
$$

The approximations (omitting the subscript 1) $d_{1}(l) \approx \tilde{d}_{1} \equiv-(l-N / 2)^{2}+N^{2} / 4$ and $d_{1}(l+2) \approx d_{1}(l)$ yield $d_{1}(l)\left(\psi_{l+2}-2 \psi_{l}+\psi_{l-2}\right) \approx 4 d_{1}(l) \partial_{l}^{2}$, which we use to write Eq. (16) as an ordinary differential equation

$$
4 \frac{E_{1}}{2} \tilde{d}_{1}(l) \partial_{l}^{2} \psi(l)+c(l)+2 \frac{E_{1}}{2} d_{1}(l) \psi(l)=E \psi(l) .
$$

We employ a change of variables, neglecting from here on $O(1 / N)$ terms, $t=l-N / 2$ and obtain

$$
\begin{gathered}
2 E_{1}\left[-t^{2}+N^{2} / 4\right] \partial_{t}^{2} \Psi(t)+\left[E_{1}\left(-t^{2}+N^{2} / 4\right)\right. \\
+c(t+N / 2)] \Psi(t)=E \Psi(t) .
\end{gathered}
$$

We identify the above equation with the Schrödinger equation of the harmonic oscillator via

$-\frac{1}{2 m} \partial_{t}^{2} \Psi(t)+\left(\frac{1}{2} m \omega(t-\mathfrak{S})^{2}+e_{\mathfrak{S}}\right) \Psi(t)=E \Psi(t)$,

with the parameters

$$
\begin{aligned}
m & =\frac{1}{-4 E_{1}\left(N^{2} / 4-t_{\mathrm{fix}}{ }^{2}\right)}, \\
\omega & =\sqrt{-E_{1}\left(C_{0}+C_{1}-D_{1}-2 E_{1}\right)\left(N^{2}-4 t_{\mathrm{fix}}{ }^{2}\right)}, \\
\mathfrak{S} & =\frac{\left(\epsilon_{0}-\epsilon_{1}\right)+\left(C_{0}-C_{1}\right)(N-1) / 2}{C_{0}+C_{1}-D_{1}-2 E_{1}}, \\
e_{\mathfrak{S}} & =E_{1} \frac{N^{2}}{4}+c_{N / 2}-\frac{1}{2} m \omega^{2} \mathfrak{S}^{2}
\end{aligned}
$$

and an energy shift $e_{\mathfrak{S}}$ independent of $t$. Note that we have fixed the contribution from the mode exchange (proportional to $\left.E_{1}\right)$ at $t=t_{\text {fix }}$, that is, taking $d_{1}\left(t_{\text {fix }}\right)$ instead of $d_{1}(t)$, in front of the derivative. With the scalings $t=T N / 2, t_{\mathrm{fix}}=T_{\mathrm{fix}} N / 2$, and $E_{1}=\frac{1}{2} C_{0}$, we then have $\mathfrak{S}=\frac{N}{6}\left(1-\frac{16}{3} X\right)$ in terms of the ratio of single-particle energy to interaction energy units $X=\epsilon_{0} / N C_{0}$.

The ground state of Eq. (19) can be solved for analytically when the absolute value of the Fock-state amplitudes $|\psi(t)|$ is considered as a continuous variable $[9,19]$

$$
|\psi(t)|=\frac{1}{\left(\pi \sigma^{2}\right)^{1 / 4}} \exp \left[-\frac{(t-\mathfrak{S})^{2}}{2 \sigma^{2}}\right] .
$$

We get the effective oscillator length of the harmonic oscillator (note that $T \in[-1,1]$ ) as

$$
\sigma^{2}=\sqrt{\frac{1}{m \omega}}=N \sqrt{\frac{2}{3}\left(1-T_{\mathrm{fix}}{ }^{2}\right)} .
$$

The single-particle-to-interaction-energy unit ratio $X$ is then calculated to be

$$
X=\frac{\epsilon_{0}}{N C_{0}}=\frac{3}{4} \frac{1}{G_{3}}\left(\Lambda+\Lambda^{5}\right) .
$$

Finally, the total energy in the continuum limit for the reduced model is given by

$$
E=\omega+e_{\mathfrak{S}}=\omega+E_{1} \frac{N^{2}}{4}+c_{N / 2}-\frac{1}{2} m \omega^{2} \mathfrak{S}^{2} .
$$

We note that, to this order, the dependence of $\sigma$ on $T_{\text {fix }}$ does not enter the continuum energy; we finally obtain, to first order in $N$,

$$
\frac{E}{N C_{0}}=\frac{N}{3}+\frac{13 N}{9} X-\frac{8 N}{27} X^{2}+O(1)
$$

Then, with $C_{0}=G_{3} \omega / N \Lambda^{3}$,

$$
\frac{E}{N \omega}=\frac{13}{12}\left(\frac{1}{\Lambda^{2}}+\Lambda^{2}\right)-\frac{\Lambda^{3}}{6 G_{3}}\left(\frac{1}{\Lambda^{2}}+\Lambda^{2}\right)^{2}+\frac{G_{3}}{3 \Lambda^{3}},
$$

which represents the continuum expression for the energy as a function of $\Lambda$, with the sole parameter $G_{3}$.

\section{B. Large coupling limit}

The minimization problem $\partial E / \partial \Lambda=0$ in the limit of Eq. (26) can be solved for real values of $G_{3}$ when $\Lambda>4.686$ 
and we get for the minimum

$$
\begin{aligned}
G_{3} & =\frac{13}{12}\left(\Lambda^{5}-\Lambda\right)+\frac{1}{12} \sqrt{193 \Lambda^{2}-482 \Lambda^{6}+\Lambda^{10}} \\
& \approx \frac{7}{6} \Lambda^{5}-\frac{127}{6} \Lambda+O\left(\frac{1}{\Lambda^{3}}\right) \approx \frac{7}{6} \Lambda^{5} .
\end{aligned}
$$

For the relative energy difference to a Fock state with all particles occupying the $l=0$ state (the radial ground state), we get

$$
\frac{\Delta E}{N \omega}=\frac{E_{\text {Fock }}-E_{\text {cont }}}{N \omega} \sim 0.00013 G_{3}^{2 / 5},
$$

with the Fock state energy

$$
\frac{E_{\mathrm{Fock}}}{N \omega}=\frac{3}{4}\left(1 / \Lambda^{2}+\Lambda^{2}\right)+\frac{G_{3}}{2 \Lambda^{3}} .
$$

At its minimum, $\Lambda_{\text {Fock,min }}^{5} \approx G_{3}$. Note also that in quasi-onedimension we have $\Delta E / N \omega \sim 0.02 G_{1}^{2 / 3}$ and in quasi-twodimension $\Delta E / N \omega_{\perp} \sim 0.002 G_{2}^{1 / 2}$ [6]. Hence, with increasing dimension, both the prefactor and the scaling of the energy difference to a single condensate decrease. For a second, fragmented minimum to exist we need $G_{3}>2436.13$ and then we have $\Delta E / N \omega \gtrsim 0.0015$.

The continuum limit is valid around the expansion point $t_{\text {fix }}$, which we set equal to the shift $T_{\text {fix }} \equiv \mathfrak{S} /(N / 2)=\frac{1}{3}-\frac{16}{9} X$. For the single-particle-to-interaction-energy ratio, we have $X=\frac{3}{4}\left(\frac{6}{7}\right)^{1 / 5} G_{3}^{-4 / 5}+\frac{9}{14}$, which asymptotically approaches $X=\frac{9}{14} \approx 0.64$ and is close to this value already for the critical $\left(G_{3}\right)_{c}=2436.13$. Then the asymptotic shift is evaluated to $\mathfrak{S} /(N / 2) \approx-0.810$, giving $\sigma^{2}=N \sqrt{\frac{2}{3}\left(1-T_{\text {fix }}{ }^{2}\right)} \approx 0.48 N$. We can now assess the validity of the continuum approach by measuring the occupation it assigns to negative (unphysical) $l_{1}$. With increasing $N$, the width of the wave function (21) gets smaller and the density at negative $l_{1}$ goes to zero as $\frac{1}{2}(1-\operatorname{erf}[0.13729 \sqrt{N}])$. The rapid convergence is illustrated for $N=1000$, when $\int_{-\infty}^{-N / 2}|\psi(t)|^{2} d t \approx 4.1 \times 10^{-10}$.

\section{Degree of fragmentation}

The degree of fragmentation in the continuum limit reads

$$
\mathcal{F}=1-\frac{2}{N} \sqrt{\left[\frac{N}{2} \sin \theta\left(1-\frac{\sigma^{2}+2 \mathfrak{S}^{2}}{N^{2}}\right)\right]^{2}+\mathfrak{S}^{2}}
$$

Here we assume that the two degenerate many-body states of the two-mode problem [9] have equal weight in the ground state and $\theta$ is their relative phase [18].
The maximal degree of fragmentation (that is, when $\theta=0$, as assumed in our numerical computations above) becomes

$$
\mathfrak{F}=\frac{4}{21}-\frac{3}{4}\left(\frac{6}{7}\right)^{1 / 5} G_{3}^{-4 / 5} .
$$

Within the validity of the continuum approximation, in the limit of large coupling, the fragmentation reaches approximately $19 \%$ and is hence significantly lower than in either quasi-1D (80 \%) and quasi-2D (33\%) trapping geometries. The power law of the asymptotics here is $4 / 5$, while in quasi-1D trapping it has been 4/3 and in quasi-2D unity [6]. This implies that the coupling dependence of the degree of fragmentation becomes weaker with increasing dimension.

Finally, we conclude from the comparison with the numerical data shown in Figs. 2 and 3 that the agreement between the two-mode continuum limit and numerics is excellent for sufficiently large values of $N$ and $G_{3}$.

\section{CONCLUSION}

A dimensionless measure $G_{D}$ of the relative importance of the total interaction and potential energies, which ultimately determines the first-order coherence properties of a trapped system, can be constructed from the three-dimensional coupling constant $g$ and the relevant trapping lengths in quasi-1D, quasi-2D, and proper three-dimensional systems. The results presented in the above, together with the quasi-1D and quasi-2D counterparts derived in Ref. [10], where we found that the critical $\left(G_{1}\right)_{c} \sim O(10)$ and $\left(G_{2}\right)_{c} \sim O(100)$, lead us to conclude that the dimensionless critical $G_{D}$ in dimension $D$, for trapped dilute Bose gases at absolute zero, scales approximately like $\left(G_{D}\right)_{c} \sim 10^{D}$ for fragmentation into two macroscopically occupied orbitals to occur. We have furthermore demonstrated that the degree of fragmentation increases more slowly with $G_{D}$ when the dimension increases.

The corollary of our result is the asymptotic irrelevance of interactions in large spatial dimensions $(D \geqslant 3)$ for the manybody phenomenon of macroscopic fragmentation to occur, due to the exponentially increasing lower bound on the critical interaction strength. The correlations leading to fragmented condensate states, which force us to go beyond the meanfield theory of a single macroscopically occupied orbital, thus become weaker with increasing spatial dimension.

\section{ACKNOWLEDGMENTS}

U.R.F. was supported by the NRF Korea (Grants No. 20100013103 and No. 2011-0029541) and the Seoul National University Foundation Research Expense. P.B. received support from the Ministerio de Ciencia e Innovación of Spain under Projects No. MTM2009-08587 and No. MTM2010-18246C03 and a FPU fellowship through Grant No. AP2009-1892.
[1] S. N. Bose, Z. Phys. 26, 178 (1924).

[2] A. Einstein, Sitzungsber. K. Preuss. Akad. Wiss. Phys. Math. Kl., Jahrgang 1924, pp. 261-267.

[3] S. de Groot, G. J. Hooyman, and C. A. ten Seldam, Proc. R. Soc. London Ser. A 203, 266 (1950); P. T. Landsberg, Proc.
Cambridge Philos. Soc. 50, 65 (1954); A. Münster, Z. Phys.

144, 197 (1956); Z. Yan, Phys. Rev. A 59, 4657 (1999).

[4] P. C. Hohenberg, Phys. Rev. 158, 383 (1967).

[5] N. D. Mermin and H. Wagner, Phys. Rev. Lett. 17, 1133 (1966).

[6] U. R. Fischer, Phys. Rev. Lett. 89, 280402 (2002). 
[7] P. Nozières and D. Saint James, J. Phys. 43, 1133 (1982).

[8] E. J. Mueller, T.-L. Ho, M. Ueda, and G. Baym, Phys. Rev. A 74, 033612 (2006).

[9] P. Bader and U. R. Fischer, Phys. Rev. Lett. 103, 060402 (2009).

[10] U. R. Fischer and P. Bader, Phys. Rev. A 82, 013607 (2010).

[11] C. Pethick and H. Smith, Bose-Einstein Condensation in Dilute Gases (Cambridge University Press, Cambridge, 2002).

[12] F. Dalfovo, S. Giorgini, L. P. Pitaevskiǔ, and S. Stringari, Rev. Mod. Phys. 71, 463 (1999).

[13] A. I. Streltsov, O. E. Alon, and L. S. Cederbaum, Phys. Rev. A 73, 063626 (2006).
[14] O. E. Alon, A. I. Streltsov, and L. S. Cederbaum, Phys. Rev. A 77, 033613 (2008).

[15] K. Sakmann, A. I. Streltsov, O. E. Alon, and L. S. Cederbaum, Phys. Rev. A 78, 023615 (2008); Phys. Rev. Lett. 103, 220601 (2009).

[16] H. Y. Lu and S. Yi, Sci. China Phys. Mech. Astron. 55, 1535 (2012).

[17] M. C. Tsatsos, A. I. Streltsov, O. E. Alon, and L. S. Cederbaum, Phys. Rev. A 82, 033613 (2010).

[18] U. R. Fischer, K.-S. Lee, and B. Xiong, Phys. Rev. A 84, 011604(R) (2011).

[19] R. W. Spekkens and J. E. Sipe, Phys. Rev. A 59, 3868 (1999). 